\title{
Differential Step Response of Unipolar Space-Charge-Limited Current in Solids
}

\author{
R. BARON \\ Hughes Research Laboratories, Malibu, California \\ AND \\ M-A. NICOLET AND V. RODRIGUEZ, \\ California Insitute of Technology, Pasadena, California
}

(Received 7 April 1966)

\begin{abstract}
The small signal step response of unipolar space-charge-limited current in solids is analyzed for planar structures and for media in which the drift velocity of the charge carriers is either proportional to the electric field (thermal charge carriers) or is independent of the electric field (hot charge carriers). Results are reported in analytical and graphical form. Their features are discussed in terms of the underlying physical phenomena, as well as in the perspective of experimental applications. Cylindrical and spherical structures are not accessible to closed-form solutions by the approach.
\end{abstract}

\section{INTRODUCTION}

$T$ HE flow of unipolar space-charge-limited current (sclc) in solids has been the object of an increasing number of investigations in recent years. Most of this work discusses primarily the steady-state conditions of the phenomenon, and only few studies are concerned with time-dependent effects. The majority of these relate specifically to the problem of the large signal turn-on transient of unipolar sclc in both theory and experiment, ${ }^{I-7}$ while the large signal turn-off transient has been studied experimentally only. ${ }^{8}$ The small signal response of unipolar sclc has received even less attention and the treatments are restricted to the frequency domain. ${ }^{9-11}$ This lack of interest is probably due to the obvious difficulties which experiments of this sort must meet. On the other hand, studies in the time domain constitute powerful tools of investigation, as amply demonstrated by the successes of the large signal turn-on investigations mentioned above and by small signal transient experiments performed on bipolar sclc. ${ }^{12}$ The present calculations have therefore been undertaken with an aim at filling this gap in the analytical coverage of unipolar sclc, thus preparing the ground for possible future experiments in this field.

The problem is treated in the Mott and Gurney approximation of scle in a solid. ${ }^{13}$ An explicit analytical

${ }^{1}$ P. Mark and W. Helfrich, J. Appl. Phys. 33, 205 (1961).

${ }^{2}$ A. Many, M. Simhony, S. Z. Weisz, and J. Levinson, J. Phys. Chem. Solids 22, 285 (1961).

${ }^{3}$ W. Helfrich and P. Mark, Z. Physik 166, 370 (1962).

4 A. Many and G. Rakavy, Phys. Rev. 126, 1980 (1962).

5. Many, S. Z. Weisz, and M. Simhony, Phys. Rev. 126, 1989 (1962).

A A. Many, M. Simhony, S. Z. Weisz, and Y. Teucher, J. Phys. Chem. Solids 25, 721 (1964).

${ }^{7}$ J. Adolph, E. Baldinger, W. Czaja, and I. Graenacher, Phys. Letters 6, 137 (1963).

${ }^{8}$ W. Helfrich and P. Mark, Z. Physik 168, 495 (1962).

9 J. Shao and G. T. Wright, Solid-State Electron. 3, 291 (1961).

${ }^{10}$ A. Shumka and M-A. Nicolet, Solid-State Electron. 7, 106 (1964).

11 G. T. Wright, Solid-State Electron. 9, 1 (1966).

12 R. Baron, O. J. Marsh, and J. W. Mayer, J. Appl. Phys. 37, 2614 (1966).

${ }_{13}$ N. F. Mott and R. W. Gurney, Electronic Processes in Ionic Crystals (Oxford University Press, Oxford, England, 1957), 2nd ed., p. 172. solution for the current response to a small voltage step is derived for a planar geometry by Laplace transform techniques. No solutions could be obtained for the cylindrical and spherical geometries by a similar procedure because in the frequency domain, integrals appear which cannot be evaluated in form of simple functions.

\section{MATHEMATICAL MODEL}

The following idealizing assumptions are made: (a) the solid is a perfect and homogeneous insulator without free charge carriers of its own or imperfections of any kind; (b) free charge carriers injected into the solid move by drift only, diffusion is negligible; (c) the drift velocity is proportional to the electric field strength; (d) the emitter maintains an infinite supply of free charge carriers at its boundary with the solid and these charge carriers possess no initial velocity of their own; (e) the collector boundary is a sink from which no free charge carriers can escape. For positive charge carriers the flow of current is then governed by the following equations:

$$
\begin{aligned}
-\operatorname{grad} \Phi & =\mathbf{E}, \\
\epsilon \operatorname{div} \mathbf{E} & =\rho, \\
\mathbf{J}_{\mathrm{dr}} & =\rho \mu \mathbf{E}, \\
\operatorname{div} \mathbf{J}_{\mathrm{dr}} & =-\partial \rho / \partial t,
\end{aligned}
$$

where $\Phi$ is the electrostatic potential, $\mathbf{E}$ is the electric field, $\rho$ is the charge density due to the mobile charge carriers, $\mathbf{J}_{\mathrm{dr}}$ is the density of the drift current, and all these quantities are functions of the space coordinates $\mathbf{x}$ and the time $t$. The dielectric constant $\epsilon$ and the mobility $\mu$ are assumed independent of $\mathbf{x}$ and $t$. Because of assumptions (b) and (d), the electric field at the emitter boundary vanishes; hence

$$
\mathbf{E}(\text { emitter, } t)=0 \text {. }
$$

The bias voltage applied across the collector and emitter is denoted by $V>0$. By eliminating $\mathbf{J}_{\mathrm{d} r}$, one obtains

$$
\operatorname{div}(\rho \mu \mathbf{E}+\epsilon \partial \mathbf{E} / \partial t)=0 .
$$


The small signal solution to this equation is found by splitting all functions into a large component independent of time $\left[\right.$ denoted by $\left.\rho_{0}(\mathbf{x}), \mathbf{E}_{0}(\mathbf{x}), \mathbf{J}_{0}(\mathbf{x}), V_{0}\right]$ and a small time-dependent component [denoted by $r(\mathbf{x}, t), \mathbf{e}(\mathbf{x}, t), j(\mathbf{x}, t), v(t)]$. This ansatz separates the steady-state equations and with their solution, retaining first-order terms only, the time-dependent part of Eq. (6) takes the form

$$
\operatorname{div}\left(\mu \rho_{0} \mathbf{e}+\epsilon \mu \mathbf{E}_{0} \operatorname{dive}+\epsilon \partial \mathbf{e} / \partial t\right)=0 .
$$

\section{ANALYSIS}

For a planar geometry, a first integration of Eq. (7) yields

$$
\mu_{\rho_{0}} e(x, t)+\epsilon \mu E_{0} \partial e(x, t) / \partial x+\epsilon \partial e(x, t) / \partial t=j(t),
$$

where $j(t)$ is the density of the current transient generated by the voltage transient $v(t)$. A Laplace transformation with respect to time converts Eq. (8) in to an ordinary differential equation

$$
\frac{d \bar{e}(x, s)}{d x}+\frac{\mu \rho_{0}+\epsilon s}{\epsilon \mu E_{0}}-\bar{e}(x, s)=\frac{\bar{j}(s)+\epsilon e(x, 0)}{\epsilon \mu E_{0}} .
$$

Instantly after the application of the small voltage step $v$, the small additional field $e(x, 0)$ is constant throughout the width $W$ of the solid, so that the initial condition is $e(x, 0)=v / W$. With the steady-state distributions

$$
\rho_{0}(x)=\left(\epsilon J_{0} / 2 \mu x\right)^{\frac{1}{2}}, \quad E_{0}(x)=\left(2 J_{0} x / \epsilon \mu\right)^{\frac{1}{2}},
$$

and the boundary condition $\bar{e}(0, s)=0$ the solution of Eq. (9) is

$$
\bar{e}(\xi, s)=\frac{j(s)+\epsilon v / W}{\epsilon s}\left(1-\frac{1}{s T_{0} \xi^{\frac{1}{2}}}+\frac{1}{s T_{0} \xi^{\frac{1}{2}}} e^{-s T_{0} \xi^{\frac{\xi}{2}}}\right),
$$

where $\xi=x / W$ is a normalized distance coordinate and $T_{0}$ is the steady-state transit time given by $\left(2 \epsilon W / \mu J_{0}\right)^{\frac{1}{2}}$. This equation is integrated once more over the width of the solid. The left-hand side yields the Laplace transform of the voltage step, which is $v / s$. Solving for $\bar{j}(s)$ then gives

$$
\bar{j}(s)=\epsilon \frac{v}{W} \cdot \frac{2 s T_{0}-2+2 e^{-s T_{0}}}{\left(s T_{0}\right)^{2}-2 s T_{0}+2-2 e^{-s T_{0}}},
$$

which is a function of $\sigma=s T_{0}$. In time units of $\tau=l / T_{0}$, the current transient is thus given by

$$
j(\tau)=\frac{\epsilon v^{\prime}}{T_{0} W} L_{\sigma}^{-1}\left[2\left(\frac{\sigma-1+e^{-\sigma}}{\sigma^{2}-2 \sigma+2-2 e^{-\sigma}}\right)\right] .
$$

The inverse transform, $L_{\sigma}{ }^{-1}$, is obtained by factoring out $2\left(\sigma-1+e^{-\sigma}\right) /\left(\sigma^{2}-2 \sigma+2\right)$, expanding the remaining part in to an infinite series, and collecting terms of equal

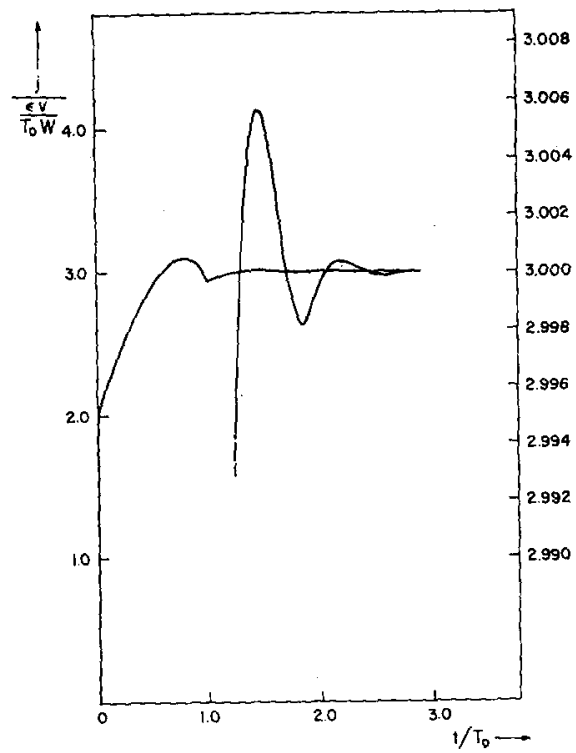

FIG. 1. Response of unipolar space-charge-limited current to a differential voltage step for a planar structure and a medium in which the drift velocity of the charge carriers is proportional to the electric field (thermal charge carriers).

powers in $e^{-\sigma}$. The final result is

$$
\left.\begin{array}{c}
j(0 \leq \tau \leq 1) /\left(\epsilon \vartheta / T_{0} W\right)=2 e^{\tau} \cos \tau \\
j(1 \leq \tau \leq 2) /\left(\epsilon \vartheta / T_{0} W\right)=2 e^{\tau} \cos \tau \\
+2 \tau e^{(\tau-1)} \sin (\tau-1) \\
j(2 \leq \tau \leq 3) /\left(\epsilon v / T_{0} W\right)=2 e^{\tau} \cos \tau \\
+2 \tau e^{(\tau-1)} \sin (\tau-1)+\tau e^{(\tau-2)} \\
\times[\sin (\tau-2)-(\tau-2) \cos (\tau-2)] \\
\vdots \quad . \quad
\end{array}\right\}
$$

This function is represented graphically in Fig. 1.

\section{DISCUSSION}

To retain only first-order terms in Eq. (7) implies that the current transient is composed everywhere of three components: the drift of the charge carriers $\rho_{0}(\mathbf{x})$ present at steady state in the additional small field $\mathbf{e}(\mathbf{x}, t)$, the drift of the additional small charge-carrier concentration $\boldsymbol{r}(\mathbf{x}, t)$ in the steady-state field $\mathbf{E}_{0}(\mathbf{x})$, and the displacement current due to the rate of change of the small additional field $\mathbf{e}(\mathbf{x}, t)$. The additional charge carriers injected into the solid after the application of the voltage step will reach the collector at $t=T_{0}$ or later. In the time interval $0 \leq t \leq T_{0}$, the current transient at the collector boundary is therefore carried only by the two components of displacement and of the steady-state charge carriers. Initially, the latter is given simply by the product $\rho_{0}$ (collector) $\mu \mathrm{e}$ (collector, 0 ) and has a value of $\epsilon v / T_{0} W$ for the planar case. The details of the calculation and Fig. 1 show that the displacement component has the same initial value, so that in this case the current transient is carried initially by two equal parts at the collector boundary. The change in the 


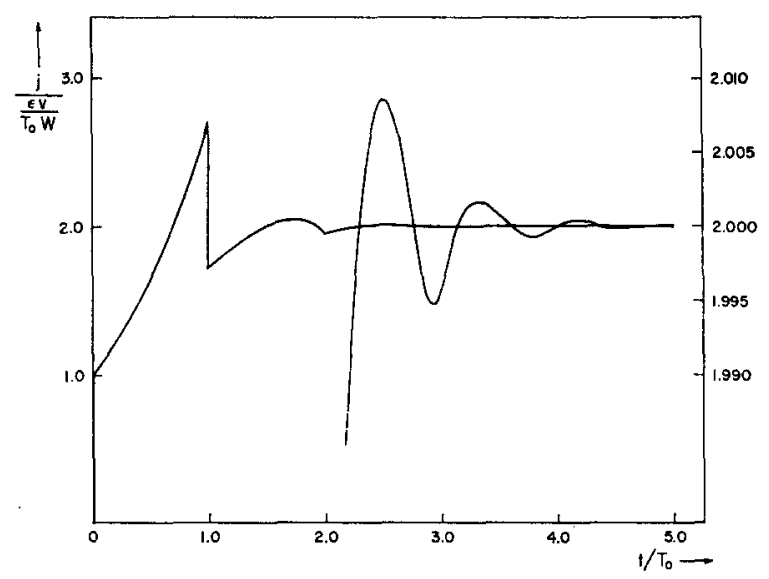

Fig. 2. Response of unipolar space-charge-limited current to a differential (and also a large) voltage step for a planar structure and a medium in which the drift velocity of the charge carriers is independent of the electric field (hot charge carriers).

form of the current response at $t=T_{0}$ indicates the first arrival at the collector of added injected charge carriers and reveals the presence of a new contribution to the current there for $i \geq T_{0}$.

Calculations along the lines outlined above have also been performed for cylindrical and spherical geometries. No explicit analytical solution could be obtained, however, because Eq. (7) leads to integrals which cannot be evaluated in form of simple functions.

Another modification of interest is that of current flow by tepid or hot charge carrier. In high mobility materials such as germanium and silicon, the assumption of a constant mobility is not valid at the high field strengths encountered in unipolar sclc. ${ }^{14,15}$ This effect can be included approximately by assuming that the drift velocity of the free charge carriers increases with the electric field $\mathbf{E}$ as $\mu_{0} E_{c}\left(\mathbf{E} / E_{c}\right)^{1 / n}, n>1$, where $\mu_{0}$ is the mobility at low fields and $E_{c}$ is a parameter. But even for the simple planar geometry, the calculations cannot be carried through to an explicit solution. If, however, attention is restricted to the limiting case of complete velocity saturation $(n \rightarrow \infty)$, Eq. (7) takes on the simple form of

$$
\operatorname{div}\left(\epsilon v_{s} \operatorname{dive}+\epsilon \partial \mathbf{e} / \partial t\right)=0,
$$

${ }^{14} \mathrm{~A}$. Shumka and M-A. Nicolet, Proceedings of the 7 th International Conference on the Physics of Semiconductors (Dunod Cie., Paris, 1964), p. 621.

${ }^{15}$ S. Denda and M-A. Nicolet, J. Appl. Phys. 37, 2412 (1966). where $v_{s}$ is the constant saturation velocity. Again, an explicit solution exists only for the planar geometry; it is

$$
\begin{gathered}
j(0 \leq \tau \leq 1) /\left(\epsilon v / T_{0} W\right)=e^{\tau}, \\
j(1 \leq \tau \leq 2) /\left(\epsilon v / T_{0} W\right)=e^{\tau}-\tau e^{(\tau-1)}, \\
j(n \leq \tau \leq n+1) /\left(\epsilon v / T_{0} W\right) \\
=\sum_{\nu=0}^{n}(-1)^{\nu}(\nu !)^{-1} \tau(\tau-\nu)^{\nu-1} e^{(\tau-\nu)} .
\end{gathered}
$$

Figure 2 shows this result graphically. The interpretation is particularly simple in this case because the field added by the application of the voltage step leaves the drift velocity of the charge carriers unchanged. The current transient thus consists of only two components, and in the time interval $0 \leq \tau \leq T_{0}$ the current response at the collector boundary is due to displacement only. The details of the solution reveal that in this time interval the impulse of charge placed at the emitter boundary at $t=0$ drifts towards the collector at the speed $v_{s}$, while more charge is following in its wake. The discontinuity at $t=T_{0}$ marks the instant at which the impulse of charge meets its counterpart at the collector boundary.

For complete velocity saturation, the time-dependent parts of the solutions of Eqs. (1)-(4) separate from the time-independent parts, whatever the magnitude of the transient. The solution hence applies for large signals as well. This property offers the possibility of a test with which velocity saturation can be detected experimentally.

The present discussion covers the current transient only in its response immediately after the application of the voltage step. $A t$ the instant of the step, a large surge of current will occur, by which the charge required in the initial conditions of the problem is provided. The actual form of this current impulse and its duration will depend critically upon the parameters of the external circuitry and are not directly related to the sclc condition under consideration. This initial phase of the current transient is therefore omitted here. But its presence and features consist a major problem in any experimental attempt to verify the present results. 